\title{
La demanda agregada de la cultura en México: propensión al consumo y tasa de interés para empresas culturales*
}

\author{
Aggregate demand for culture in Mexico: propensity to consume and interest rates of \\ cultural enterprises
}

\author{
Pablo Sigfrido Corte-Cruz \\ Doctor en Economía, Benemérita Universidad Autónoma de Puebla, \\ Puebla de Zaragoza, México, pablo.corte@correo.buap.mx
}

\begin{abstract}
Cómo citar / How to cite
Corte-Cruz, P. S. (2019). La demanda agregada de la cultura en México: propensión al consumo y tasa de interés para empresas culturales. Revista CEA, 5(10), 151-165. https://doi.org/10.22430/24223182.1331
\end{abstract}

Recibido: 15 de abril de 2019

Aceptado: 30 de junio de 2019

\section{Resumen}

El presente trabajo es un ejercicio macroeconómico a partir de la información del Sistema de Cuentas Satelitales de la Cultura de México, que publica el Instituto Nacional de Estadística y Geografía (INEGI), considerando la contabilidad económica en el ramo. El objetivo es determinar un modelo de demanda agregada del sector de la cultura, a partir del cálculo de una propensión marginal al consumo y de una tasa de interés para empresas culturales. Para lograr la meta, se considera la literatura macroeconómica tradicional para deducir el gasto autónomo propio del ramo y, con ello, la curva de bienes culturales. También se toma en cuenta la presencia de la curva del mercado de dinero para obtener el propósito señalado. Se utilizan Mínimos Cuadrados Ordinarios (MCO) y, en el caso de presencia de heterocedasticidad, Mínimos Cuadrados Ponderados (MCP). Se concluye que la población mexicana, en promedio, no gasta más allá del $5.3 \%$ de sus ingresos en bienes culturales y que las empresas creativas, por sus mismas condiciones, requieren de tasas de interés muy por debajo de las del total del mercado financiero.

Palabras clave: cultura y desarrollo, economía cultural, planificación cultural, autonomización.

\begin{abstract}
This work is a macroeconomic exercise based on information in the Mexican Culture Satellite Account published by the National Institute of Statistics and Geography. Its objective is to determine an aggregate demand model of the cultural sector in Mexico based on calculations of its marginal propensity to consume and interest rates. Traditional macroeconomic literature was considered to reduce the autonomous spending of the sector and, therefore, the curve of cultural assets. The
\end{abstract}

* Este artículo se deriva del proyecto titulado «La demanda agregada de la cultura en México: propensión al consumo y tasa de interés para empresas culturales» y ha sido financiado con recursos propios. 
presence of the curve of the money market was also taken into account to achieve such objective. Ordinary Least Squares (OLS) was used and, in presence of heteroscedasticity, Weighted Least Squares (WLS) was implemented. In conclusion, average Mexicans do not spend more than 5.3\% of their income in cultural goods, and creative industries, because of their conditions, require interest rates well below those of the general financial market.

Keywords: Culture and development, cultural economy, cultural planning, autonomization.

\section{INTRODUCCIÓN}

La economía de la cultura tiene como fin estudiar el comportamiento en áreas de competencia de las empresas culturales, pero también implica un sentido de política pública que permite el acercamiento al consumidor; es decir, al público, ya sea desde lo material (un foro, teatro, vía pública) o inmaterial (los procesos artesanales, usos y costumbres, música folklórica, entre otros) (Aguado Quintero, Palma y Pulido Pavón, 2017). Tiene vínculos muy estrechos con la economía conductual pues se requiere del desarrollo de empresas culturales y la generación de consumidores de este tipo de bien, forjando propuestas que beneficien, tanto al que invierte en este tipo de eventos como a los trabajadores de la cultura, lo cual contrae una cuestión propia de la microeconomía (Monasterio Astobiza, 2017).

Pero al considerar este comportamiento desde una visión agregada, donde se revisa desde los gastos de los consumidores privados, así como desde las mismas autoridades políticas, implica una revisión sobre los conceptos de inversión de las empresas culturales y sus relaciones comerciales con el exterior, lo que conlleva un análisis macroeconómico.

El presente documento, es un ejercicio a partir de la información del Sistema de Cuentas Satelitales de la Cultura en México (CSC) que publica el Instituto Nacional de Estadística y Geografía (INEGI) (2018a), con respecto a la contabilidad económica en el ramo, basado en la literatura macroeconómica elemental sobre la demanda agregada, el consumo, la inversión y el gasto autónomo, sobre bienes y empresas culturales. La finalidad de este ejercicio es determinar la propensión al consumo que tienen los mexicanos en bienes culturales y calcular la tasa de interés que debe pagar este sector para el último año de este trabajo.

\section{MARCO TEÓRICO}

Al establecer criterios sobre la cultura y su influencia en la economía, se han establecido un conjunto de principios para su análisis y revisión, la mayor parte desde el marco microeconómico de la economía conductual. Por lo mismo, los estudios sobre la Economía de la Cultura han remarcado su importancia considerando las diversas perspectivas de los saberes económicos (Cooter \& Ulen, 2016).

Según Throsby (2001), es una parte del estudio de la sostenibilidad, por lo que las esferas, social, económica y ambiental, siempre serán vinculadas a la cultura, a decir: dado que uno de las principales connotaciones de la sostenibilidad hace referencia al entorno natural y a su relación con los procesos económicos, y, dadas las situaciones entre el entorno natural y cultural y entre el capital natural y 
cultural, se puede sugerir fácilmente que las interpretaciones ambientales de la Sostenibilidad tendrán un equivalente en la esfera de la cultura.

De acuerdo a Bourdieu (2011), la cultura es parte de los procesos de reproducción social y una de las partes determinantes de los modos de vida, de ahí que establece tres estados del capital cultural, además, en el estado incorporado, es decir, bajo la forma de disposiciones duraderas del organismo; en el estado objetivado, bajo la forma de bienes culturales, cuadros, libros, diccionarios, instrumentos, maquinaria, los cuales son la huella o la realización de teorías o de críticas a dichas teorías, y de problemáticas, etc., y finalmente en el estado institucionalizado, como forma de objetivación muy particular, porque tal como se puede ver con el título escolar, confiere al capital cultural -que supuestamente debe de garantizar - las propiedades totalmente originales.

En los últimos años, la política cultural se ha debilitado por la falta de estrategias económicas y sociales, este desdeño es producto de la poca importancia que tiene el tema de la cultura en diferentes ámbitos políticos, reflejada principalmente por una disminución del presupuesto destinado a la cobertura de proyectos culturales, por lo cual, no satisface las necesidades de la población a nivel educativo y cultural. Con ello se evidencia una decadencia en la generación de conciencia intelectual a nivel nacional, estatal o municipal (Molina Roldán, 2016).

Al buscar una definición de Economía de la Cultura, se localiza a Palma \& Aguado (2010) que, al retomar las ideas de Mark Blaug, mencionan que la formación de gustos en las artes, oferta y demanda de bienes y servicios culturales, industria de las comunicaciones, mercado del arte, historia económica de las artes, mercado de trabajo de los artistas, enfermedad de los costos, organizaciones artísticas sin ánimo de lucro y subsidios públicos a las artes.

También existen algunos autores que determinan alguna corriente de pensamiento económico, tal y como menciona Batista (2006) que la economía cultural contribuye así a hacer visibles o rescatar otras visiones de lo económico ignoradas, ocultadas o suprimidas, directa o indirectamente, por la economía. La segunda se ocuparía de las concepciones que mantienen y difunden los investigadores de la economía, fundamentalmente los economistas. En este caso, y como se dijo antes, la economía se antropologiza, se vuelve materia de la reflexión y la crítica antropológicas.

La economía de la cultura se puede analizar desde dos posturas: las tendencias artísticas como el multiculturalismo que subrayan la justicia social (entendida de un modo estrecho como una representación visual equitativa en las esferas públicas) y las iniciativas para promover la utilidad sociopolítica y económica se fusionaron (Yúdice, 2002).

Sin embargo, no sólo existen estas dos corrientes para entender y renovar las diferentes ramas de la Economía; otra visión es propuesta en el Informe sobre la Economía Creativa 2013, elaborado por la organización de las Naciones Unidas para la Educación, la Ciencia y la Cultura (Unesco): "En un momento en el que los países están tratando de alcanzar los Objetivos de Desarrollo del Milenio y el mundo está diseñando la nueva Agenda de Desarrollo Global Post-2015, se debe reconocer la importancia y el poder de los sectores creativos y culturales como impulsores de desarrollo humano sostenible". 
De acuerdo a lo mencionado en dicho Informe, el punto principal es incluir en el Producto Interno Bruto (PIB) de todos los países la participación de los actores sociales en relación al entorno cultural, como parte del desarrollo económico. Es decir, "En la dimensión economía se analiza la contribución del sector cultural al desarrollo económico mediante el examen de tres indicadores centrales: la contribución de las actividades culturales al PIB, el empleo cultural y el gasto de los hogares en cultural" (Unesco, 2014).

Por tanto, la cultura también contiene un valor económico que, en muchas ocasiones, no ha sido considerado, tanto por los que realizan políticas culturales, así como los hacedores de política económica. En ese sentido Achugar (2000), citado por Guzmán Cárdenas (2003) afirma que:

\begin{abstract}
La investigación y el análisis de la relación entre cultura, valor y trabajo [...] han sido, si no nulos, bastante escasos y, en el mejor de los casos, han sido realizados, desde presupuestos teóricos y disciplinarios [...] como una parte menor de investigaciones cuyos intereses no estaban centrados en la elaboración de políticas públicas de la cultura. Esto se debe a varias razones, pero entre las fundamentales es posible enumerar: 1) la persistencia en la sociedad [...] de una concepción acerca de la cultura que entiende que el 'valor' cultural es simbólico y, por lo mismo, redituable sólo a nivel espiritual, así como la de una concepción 'demonizada' de la 'cultura masiva' y de las llamadas 'industrias culturales', 2) la ausencia de interés por la 'economía de la cultura' tanto en los encargados de elaborar y administrar políticas culturales como entre los economistas [...] y la consecuente ausencia de dicha problemática en los planes de estudio de las universidades [...]; y en parte, en función de lo anterior: 3 ) la ausencia de datos desagregados relativos a los bienes y servicios culturales en los informes y estadísticas suministrados por las reparticiones estatales (pp. 7-8).
\end{abstract}

Ahora bien, y considerando las recomendaciones de la Unesco, México inicia la publicación de las Cuentas Satelitales de la Cultura (CSC), las cuales, son elaboradas por el Instituto Nacional de Estadística y Geografía (INEGI) (2018b) y la Secretaría de Cultura, cuya finalidad es integrar la información de todas las actividades culturales que participan en la economía mexicana.

Con lo anterior, también se han creado las Estadísticas Básicas de la Cultura en México, elaboradas de la misma forma por la ahora Secretaría de Cultura y estableciendo las principales variables como: patrimonio, infraestructura, medios impresos, cine, radio, televisión, nuevas tecnologías, equipamiento en viviendas, prácticas culturales y tiempo libre, entre otras.

Es evidente que hay poco sustento teórico-académico con referencia a la Economía de la Cultura, teniendo como fuente algunos trabajos escritos tanto electrónico como en forma física. Así como la incorporación de artículos científicos y alguna información hemerográfica, sin incluir la perspectiva de desarrollo sostenible o sustentable.

Se tiene como antecedente la Encuesta Nacional de Hábitos, Prácticas y Consumo Culturales, que publicó el Consejo Nacional de las Culturas y las Artes (Conaculta, 2010), ahora Secretaría de Cultura. En ella se establece que, en promedio, el $64.5 \%$ de la población mexicana no gasta en bienes y servicios culturales. Este dato indica que, por un lado, la mayor parte de la gente no está interesada en la cultura y, por otro lado, la gente busca actividades artísticas de bajo costo, o incluso de manera gratuita. Este último dato implica que la inversión realizada en publicación en libros y la producción de música, presentaciones de danza clásica y tradicional, artes audiovisuales, artes plásticas y teatro, 
no se vea con rendimientos adecuados, más si estos requieren de algún crédito con intereses para lo mismo.

\subsection{Revisión macroeconómica básica}

Para determinar un modelo a partir de las CSC, primero se debe de considerar el cálculo del Producto Interno Bruto (PIB), tanto nacional, como uno propio para la contabilidad de la cultura. De ahí que hay que remontarse a la literatura básica de la Teoría económica, especialmente al texto de Dornbusch, Fischer \& Startz (2004).

Considerando la contabilidad del gasto, el PIB $(Y)$ se determina a partir del consumo privado de las familias $(C)$, el consumo del gobierno $(G)$, la inversión bruta en capital fijo $(I)$ y la balanza comercial neta $(X N)$, producto de restar la demanda de bienes externos de la demanda de bienes internos por parte del exterior

$$
Y=C+G+I+X N
$$

Si bien esta fórmula es de conocimiento muy básico, es fundamental para considerar, tanto al PIB total como el cultural, al mismo tiempo que a la identidad del consumo

$$
C=C A+c^{*} Y D
$$

donde $C A$ es el consumo autónomo, YD es el ingreso disponible y c es la propensión al consumo.

Según Blanchard, Amighini \& Giavazzi (2012), el YD es lo que queda del ingreso después de pagar los impuestos $(T)$, siendo esta una manera muy sencilla de calcularlo

$$
Y D=Y-T
$$

Para la deducción matemática de una propensión al consumo en cultura, se mantiene el valor total de la economía en el YD. La fuente estadística de los impuestos es la página electrónica del Banco Mundial (s.f.), considerando únicamente al referente al pago en bienes y servicios, los cuales han aumentado del $21.8 \%$ al $30.4 \%$ en el lapso de estudio (del 2008 al 2017).

Otra entidad importante para este ejercicio macroeconómico es la referente a la inversión, la cual está determinada, principalmente, por la tasa de interés.

$$
I=I A+b * i
$$

donde IA es la inversión autónoma, $i$ es la tasa de interés y $b$ es la pendiente de la curva de inversión. Para el caso de la tasa de interés, se determina la Tasa de Interés Interbancaria de Equilibrio (TIIE) que es la tasa activa promedio para los créditos de las entidades bancarias, teniendo como fuente al INEGI (2018a).

Con respecto al cálculo del gasto autónomo $(G A)$, se considera el supuesto de que $G$ y $X N$ son totalmente autónomos, mientras que, para el consumo y la inversión autónomos, se determinan a partir de las identidades (2) y (4) respectivamente. 
Con base en lo anterior se construye la curva de mercado de bienes culturales, considerando el efecto multiplicador $(\alpha)$, el cual se calcula con la propensión al consumo en cultura determinado por (2) y la tasa de impuestos promedio en bienes y servicios $(t)$

$$
\begin{aligned}
& \alpha=\frac{1}{1-c(1-t)} \\
& Y=\alpha\left(G A-b^{*} i\right)
\end{aligned}
$$

La demanda agregada (DA) no sólo considera el equilibrio del mercado de bienes, también supone que el mercado de dinero se encuentra en equilibrio, en el cual la oferta de saldos reales $(M / P)$ es igual a la demanda de circulante $(L)$.

$$
\frac{M}{P}=L(i, Y)
$$

Con lo anterior, Dornbusch, Fischer y Startz (2004) desarrollan el modelo completo para DA, considerando la intersección de ambos mercados, en los cuales se determina una tasa de interés de equilibrio a partir del GA y la oferta real de dinero.

$$
i=\lambda G A+\delta \frac{M}{P}
$$

donde $\lambda$ es un parámetro de política fiscal, mientras que $\delta$ es el correspondiente a la política monetaria.

En ese sentido, para la realización de este ejercicio se consideran los valores otorgados por el INEGI (2018a) en el sistema de CSC, partiendo de las identidades ya señaladas, basados en los conceptos básicos de la literatura macroeconómica básica.

\section{METOdOLOGÍA}

\subsection{Estimaciones}

Para la determinación de la propensión al consumo de la cultura, en primer lugar, se considera a la identidad (2), en la cual se construye a partir del YD total de la economía:

$$
C C=\beta 0+\beta 1 Y D
$$

donde $\mathrm{CC}$ es el gasto privado en cultura, $\beta 0$ es el consumo autónomo y $\beta 1$ es la propensión al consumo en cultura.

La regresión para la inversión considera el papel de la tasa de interés, en este caso, la TIIE, pues toda empresa se acerca a las instituciones de crédito, incluso las empresas culturales, para poder invertir en sus actividades.

$$
I C=\beta 0+\beta 1 T I I E
$$


donde IC es la inversión bruta en cultura. Aquí $\beta 0$ es la inversión autónoma y $\beta 1$ es la pendiente de la curva de inversión. Con el valor constante, al igual que el del resultado de (2a), se considera para la construcción del GA para cultura.

Para la construcción del mercado de bienes culturales, primero se deduce el efecto multiplicador de la cultura a partir de las identidades (5) y (6), considerando la propensión al consumo a la cultura calculada en (2a) y el promedio de la tasa de impuestos del periodo de estudios.

Considerando por dado el equilibrio del mercado real del dinero de la identidad (7) y considerando que la importancia de este ejercicio es la determinación de una tasa de interés para empresas culturales, se regresa el modelo:

$$
\mathrm{TIIE}=\beta 0+\beta 1 \mathrm{GAC}+\beta 2 " \mathrm{M} " / \mathrm{P}^{\prime \prime}
$$

donde GAC es el gasto agregado de la cultura que se construye de los resultados de (2), (4) y los gastos de gobierno y exportaciones netas culturales.

Se considera que este resultado debe ser comparado con las tasas de interés de mercado para determinar si se deben otorgar intereses especiales al sector de la cultura, en primer lugar, porque en México, son pocos los que cuenta con una holgura de recursos monetarios para publicar y/o producir bienes culturales de manera independiente.

Los resultados, tanto de (2a) como de (8a), otorgan, por un lado, la disposición a gastar por parte de los consumidores $y$, por otro, la capacidad de producir a ciertas tasas de interés, considerando que muy poca gente gasta en bienes y servicios culturales, lo que complica los rendimientos de quienes invierten en publicación y producción.

\subsubsection{Tratamiento de los datos}

La información estadística con la que se trabaja, se obtiene del Sistema de Cuentas Satelitales de la Cultura (CSC) que publica el INEGI (2018a) en su página electrónica, con excepción del Producto Interno Bruto Total e Impuestos, que se ubican en el Banco de Información Económica (BIE). Todos los datos se encuentran en valores reales a precios de 2013.

Las CSC se ubican en datos anuales por lo que se convierten, primero a trimestrales, a partir del índice de precios del PIB. Al tener toda la información trimestral, nuevamente se realiza una conversión considerando al Indicador Global de Actividad Económica (IGAE), de acuerdo a la propuesta que realiza Elizondo (2012), quien demuestra que el diferencial de los logaritmos, tanto del IGAE como del PIB se acercan demasiado.

Con respecto a la información de la Tasa de Interés Interbancario de Equilibrio (TIIE) y la oferta monetaria, también se localizan en el BIE, las cuales tienen una periodicidad mensual. Con respecto a la última variable mencionada, al encontrarse en términos nominales, se tuvo que deflactar con respecto al índice de precios al consumidor para obtener valores reales. 
La determinación del PIB de la cultura y del Ingreso Disponible (YD) para la cultura, se siguen las identidades (1) y (3) respectivamente.

\section{RESULTADOS}

El papel de la cultura en la economía mexicana ha sido relevante en los últimos años, en especial, porque se incrementa su grado de participación en la conformación del Producto Interno Bruto (PIB), pasando del $2.9 \%$ al $3.2 \%$ del 2008 al 2017, lo cual representa una importancia similar a la de todo el sector primario que se mantiene el mismo nivel de aportación. Esto implica una tasa de crecimiento anual del 4.2 \% para este sector; es decir, dos veces más que del total del valor de los bienes producidos y realizados al interior del país.

En la Figura 1, se muestra el crecimiento anual de la cultura en comparación del total de la economía. Se verifica que, en muchas ocasiones, el crecimiento del valor de las actividades, bienes y servicios culturales se encuentra muy por arriba del crecimiento del PIB, pero también implica una tasa anual por encima del sector primario, el cual cabe mencionar, tiene una tasa de crecimiento anual del $1.96 \%$, durante este periodo.

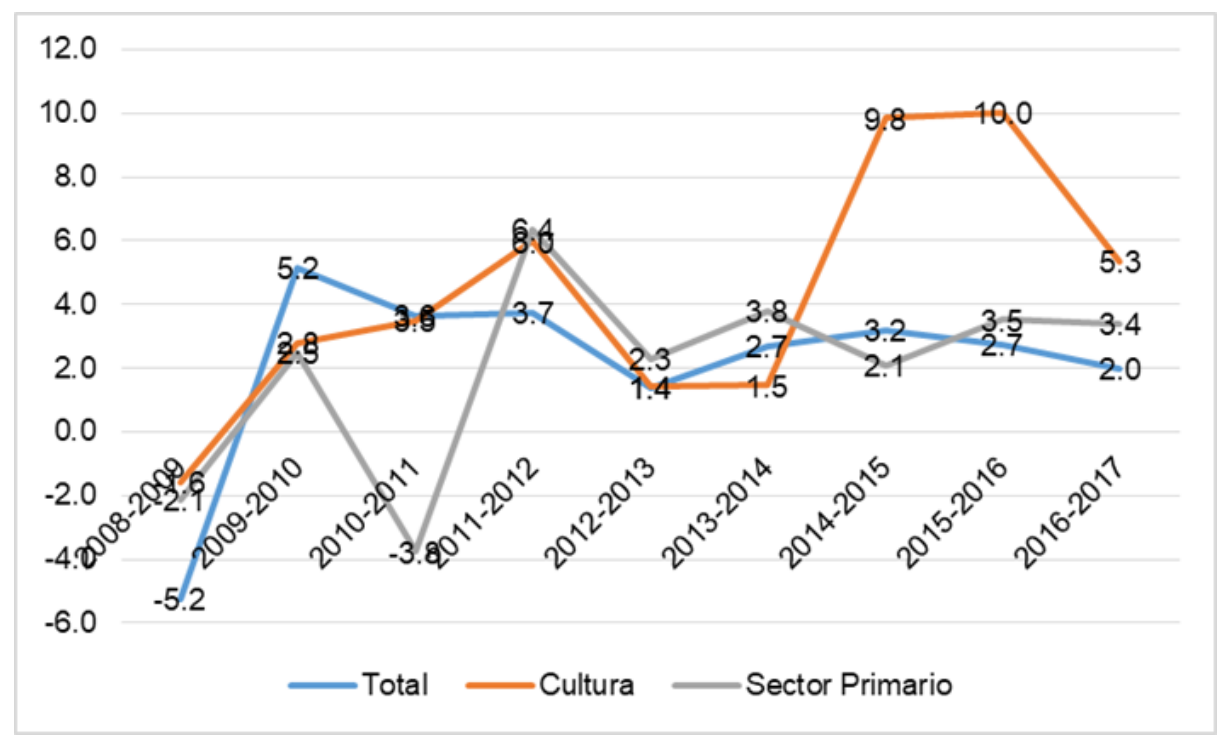

Figura 1. Tasas de crecimiento económico anual 2008-2017

Figure 1. Annual economic growth rate 2008-2017

Fuente: elaboración propia a partir del Sistema de Cuentas Satelitales de la Cultura e Indicadores Económicos de Coyuntura del Banco de Información Económica (BIE) INEGI (2018b).

Esto implica una perspectiva para considerar a la cultura como parte del ejercicio macroeconómico que aquí se presenta, puesto que la dinámica de este sector implica que existe un consumo y oferta crecientes, aún por encima del total del sector primario.

Considerando los resultados de la regresión (2a), se toma en cuenta el YD total de la economía, el mismo que tienen el total de los agentes agregados de la economía, del cual ha sido deducido, como ya se ha mencionado, del ingreso total de la economía después del pago de impuestos en bienes y servicios, según los datos del Banco Mundial. Los resultados se muestran en la Tabla 1, en el cual se 
muestra, que el estimador correspondiente al Consumo Autónoma de la Cultura, no es estadísticamente significativo.

Se destaca que ambos casos se aplican Mínimos Cuadrados Ponderados para corregir la heterocedasticidad, en la columna (1) hay presencia de autocorrelación, mismo que ya no aparece en (2), de acuerdo a la prueba Durbin-Watson.

Tabla 1. Resultados en los ejercicios planteados sobre comportamientos Table 1. Calculation of spending in culture

\begin{tabular}{|c|c|c|}
\hline \multicolumn{3}{|c|}{ Variable dependiente: consumo en cultura } \\
\hline & $(1)$ & $(2)$ \\
\hline \multirow{3}{*}{ Ingreso disponible } & $0.0627^{*}$ & $0.0526 *$ \\
\hline & $(0.0085)$ & $(.0005)$ \\
\hline & [6.87] & [102.04] \\
\hline \multirow{3}{*}{ Consumo autónomo } & -119760 & \\
\hline & $(61297.54)$ & \\
\hline & [-1.96] & \\
\hline $\mathrm{R} 2$ & 0.3699 & 0.9889 \\
\hline $\mathrm{n}$ & 120 & 120 \\
\hline
\end{tabular}

Fuente: elaboración propia a partir del Sistema de Cuentas Satelitales de la Cultura (INEGI) INEGI (2018b).

Posteriormente se considera el resultado de estas regresiones para la determinación del efecto multiplicador y el GA. La función de inversión (4a), presenta el problema de la heterocedasticidad por lo que se tuvo que recurrir a los Mínimos Cuadrados Ponderados (MCP) para poder obtener Mejores Estimados Linealmente Insesgados (MELI). Los resultados que se muestran en la segunda columna de la Tabla 2, ya implica un resultado más satisfactorio, tanto para la construcción del GA, como del mercado de bienes culturales y la tasa de interés a partir de la identidad de la demanda agregada.

Tabla 2. Determinación de la inversión en cultura

Table 2. Calculation of investment in culture

\begin{tabular}{ccc}
\hline \multicolumn{3}{c}{ Table 2. Calculation of investment in culture } \\
\multicolumn{3}{c}{ Variable dependiente: inversión en cultura } \\
\hline & MCO & MCP \\
\hline \multirow{2}{*}{ TIIE } & $-280.9421^{*}$ & $-307.912^{*}$ \\
& $(71.976)$ & $(41.918)$ \\
& {$[-3.90]$} & {$[-7.35]$} \\
\hline \multirow{3}{*}{ Inversión autónoma } & $23357.97^{*}$ & $23501.88^{*}$ \\
& $(386.257)$ & $(296.012)$ \\
\hline R2 & {$[60.47]$} & {$[79.40]$} \\
\hline $\mathrm{n}$ & 0.1144 & 0.1702 \\
\hline
\end{tabular}

* Significancia del 0.01

Fuente: elaboración propia a partir del Sistema de Cuentas Satelitales de la Cultura (INEGI) INEGI (2018b). 
Resulta interesante ver que ante un aumento del $1 \%$ de la tasa de interés, la inversión en cultura cae en 307.912 millones de pesos; es decir, este rubro representa casi el $1 \%$ del total cuando esta disminución es de 30 mil millones del total de la economía durante el periodo de estudio. De igual manera se aplica la prueba Durbin-Watson para probar que no existe la autocorrelación.

Con estos resultados se calcula el efecto multiplicador de la Economía de la Cultura, considerando el promedio de la tasa de impuestos en bienes y servicios del periodo de estudios, el cual es del $25.57 \%$. Así este efecto es de:

$$
\alpha=\frac{1}{1-0.0526(1-0.2557)}=1.0407
$$

De igual manera, considerando a $G$ y $X N$ autónomos y, de acuerdo a los resultados mostrados en la Tabla 2, se construye el GAC. Debe adicionarse que no se toma en cuenta al CA de cultura al no ser estadísticamente significativo. Así, el mercado de bienes culturales, se construye acorde a la identidad (6) y cuyo resultado se muestra en la Figura 2.

Si bien no muestra nada nuevo, a diferencia de lo que es la curva IS de los libros de texto sobre macroeconomía, aquí se considera sólo el comportamiento general de la cultura, y su construcción sólo es una verificación de comportamiento de acuerdo a las lecturas consideradas.

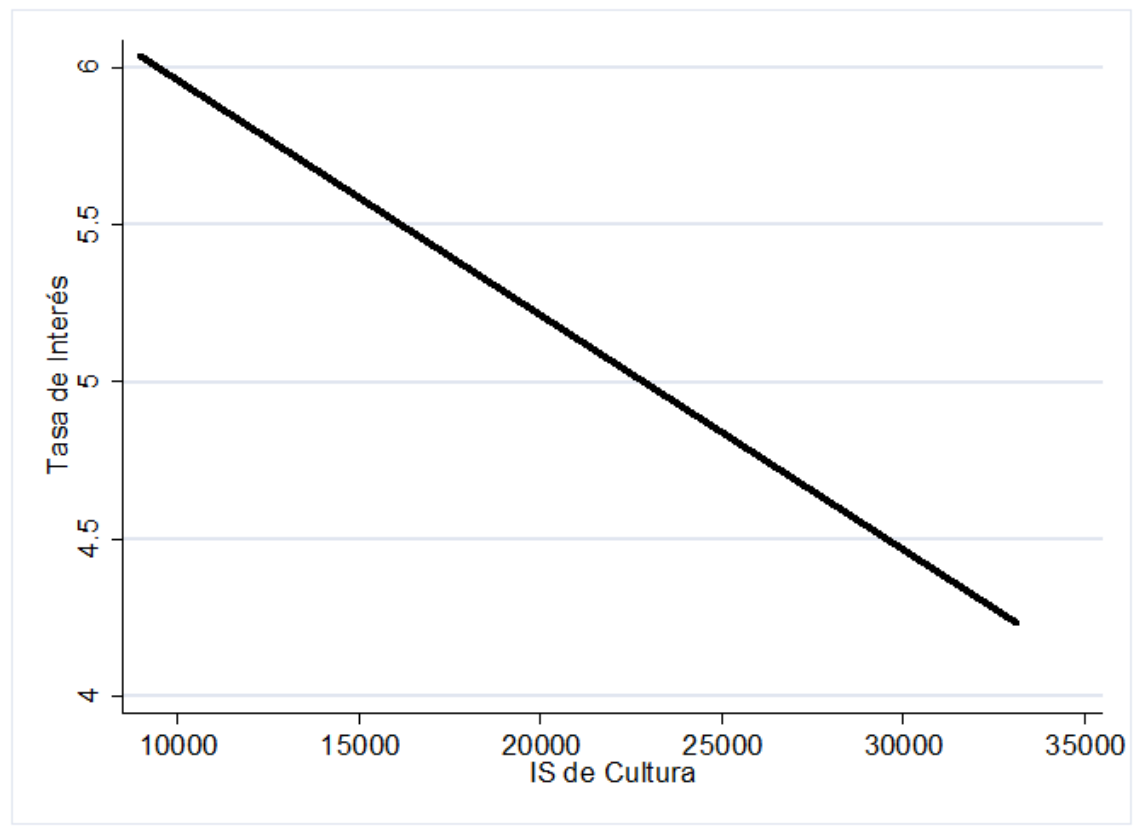

Figura 2. Estimación del mercado de bienes culturales

Figure 2. Estimation of the market of cultural goods

Fuente: elaboración propia a partir del Sistema de Cuentas Satelitales de la Cultura e Indicadores Económicos de Coyuntura (INEGI) del Banco de Información Económica (BIE) INEGI (2018b).

A partir de esto se considera el papel tanto del GA como del valor real del dinero para determinar una tasa de interés especial para el sector de la cultura. 
Si bien la propuesta de Dornbusch, Fischer y Startz (2004) es establecer una tasa de interés desde una función de demanda agregada (donde los mercados de bienes y de dinero se encuentran en un nivel de equilibrio), su cálculo para cultura debe ser una propuesta tal y como se muestra en la Tabla 3.

Tabla 3. Determinación de la tasa de interés a partir de la demanda agregada de la cultura Table 3. Calculation of interest rate based on aggregate demand for culture

\begin{tabular}{|c|c|c|}
\hline \multicolumn{3}{|c|}{ Variable dependiente: tasa de interés } \\
\hline & $\mathrm{MCO}$ & MCP \\
\hline \multirow{3}{*}{ Gasto agregado en cultura } & $--0.0002^{*}$ & $-0.0003 *$ \\
\hline & $(0.000)$ & $(0.000)$ \\
\hline & {$[-6.85]$} & {$[-10.76]$} \\
\hline \multirow{3}{*}{ Dinero (valor real) } & $0.00001^{*}$ & $0.00001^{*}$ \\
\hline & $(0.000)$ & $(0.000)$ \\
\hline & [5.15] & {$[6.56]$} \\
\hline \multirow{3}{*}{ Constante } & $4.3167^{*}$ & $5.7389 *$ \\
\hline & $(0.565)$ & $(0.4431)$ \\
\hline & {$[7.64]$} & [12.95] \\
\hline $\mathrm{R} 2$ & 0.3111 & 0.5537 \\
\hline $\mathrm{n}$ & 120 & 120 \\
\hline \multicolumn{3}{|c|}{ () Errores estándar, [] t- Estadístico } \\
\hline
\end{tabular}

Si bien los coeficientes estimados son pequeños, debe recordarse que los valores monetarios se ubican en millones de pesos a precios constantes al valor del dinero en 2013. Cabe destacar, nuevamente, que no hay presencia de autocorrelación.

Así, la tasa de interés calculada para el sector, durante el año 2017, considerando los promedios de GA y de los billetes y monedas en circulación, debiera haber sido de aproximadamente del 5.09\%, cuando esta representaba alrededor del $7 \%$ en el total de la economía.

Considerando que muchas compañías, editoriales, productores musicales independientes, entre otros, se endeudan con instituciones de créditos, se deberían formular intereses particulares, ya que es difícil tener rendimientos por las actividades culturales que llevan a cabo y sin apoyos públicos.

\section{DISCUSIÓN}

Se ha marcado en la literatura tradicional que el crecimiento económico trae educación y cultura. Sin embargo, a pesar de que el valor económico de la cultura presenta tasas de crecimiento por encima del PIB, los resultados no favorecen a la demanda por actividades, bienes y servicios culturales.

La propensión al consumo de la Tabla 1 , señala que 0.0526 centavos por cada peso del ingreso disponible se destina al gasto en cultura. Si bien la gran parte del ingreso disponible se gasta en artículos de primera necesidad, este resultado también señala que el gasto en bienes y servicios culturales no es un aspecto que, en primer lugar, la mayoría de las personas considere como una necesidad y, en segundo lugar, que se busca en un bajo costo e, incluso, de manera gratuita. 
En otros términos, la gente le apuesta a consumir productos de cultura masiva debido a muchas razones, como la pertenencia a grupos sociales cercanos, los medios de comunicación con la poca intensión de transmitir programas culturales, poca difusión por parte de las políticas públicas, la denominación de gratuidad de los eventos artísticos e, incluso, la propagación de la idea que la cultura es exclusiva para las 'personas estudiadas' o de 'alto nivel' socioeconómico. Estos resultados parecen dar la razón a Achugar 2000 (citado por Guzmán Cárdenas, 2016), en el sentido que el valor de la cultura no es económico, sino más bien, "simbólico". De ahí, la poca disposición a pagar por bienes y servicios culturales, puesto que su supuesta promoción, no promueve realmente su consumo a través de su gasto.

La inversión en cultura representa sólo el 1 \% del total de la inversión bruta fija de la economía mexicana. Esto implica, como ya ha mencionado Achugar 2000 (citado por Guzmán Cárdenas, 2016), la falta de interés real de crear políticas culturales con los artistas y desde una perspectiva económica. Políticas no asistencialistas (como los llamados Fondos a la Cultura) y que beneficien a todos aquellos que invierten en proyectos que les generen rendimientos.

La construcción del mercado de bienes culturales y de una demanda agregada de la cultura, contrae un sentido de estudio de comportamiento económico. El resultado no difiere de lo que señalan la literatura básica sobre macroeconomía y, sin embargo, también trae resultados interesantes para el sector de la cultura.

Así, la determinación de una tasa de interés por debajo de los rendimientos del mercado, hace menos pesada la carga financiera para los que invierten en proyectos culturales y es un mecanismo de política a favor de los artistas.

Con esto, las políticas culturales no sólo deben ser de inversión, sino también de consumo, para que esta promoción implique mayores rendimientos a los artistas. Las políticas deben estar fundamentadas en la idea que su promoción, inversión y consumo, mejora las condiciones intelectuales, sociales y económicas de la población.

Juntando la idea de Achugar 2000 (citado por Guzmán Cárdenas, 2016) con la de Batista Medina (2006) acerca de que el análisis económico de la cultura despliega una serie de condiciones que han sido ignoradas por los economistas, abre una pregunta: ¿hay que asumir una sola tasa de interés o debe existir una por cada sector de la economía? Por lo menos, debe existir un análisis de política económica más serio, lo que supone una disposición para acercar créditos al sector cultural, por ejemplo, una política de tasas de crédito que puedan ser subsidiadas desde el aparato gubernamental.

\section{CONCLUSIONES}

Este trabajo es un ejercicio macroeconómico para construir una demanda agregada propia de la cultura, partiendo de las identidades presentadas en la literatura macroeconómica elemental y, con la información establecida en el Sistema de CSC que publica el INEGI (2018a), para el caso de México.

Para trabajar este documento, de carácter exploratorio, se revisan algunas referencias que contextualizan el papel de la cultura en el aspecto social, como lo es dentro del campo de la 
sostenibilidad y los modos de vida, para posteriormente remarcar el desarrollo del concepto de la Economía de la Cultura, así como el valor económico de la misma.

El papel económico de la cultura ha establecido el desarrollo de las CSC, con las que se fundamenta el desarrollo del presente trabajo.

La Economía de la Cultura trae una relevancia en México, por el papel que se señala, dejando que se puedan desarrollar nuevas propuestas de política económica en este rubro.

Al tener una información anual, se tuvo que partir del desarrollo del PIB trimestral, a partir de su índice de precios, para convertir la información trimestral, y de ahí se considera al IGAE para su conversión mensual.

La aplicación de modelos MCO y MCP consideran las pruebas existentes, no sólo con respecto a la posible presencia de heterocedasticidad, sino también probar, en cada caso, si existe o no la autocorrelación serial para que los estimadores puedan ser MELI.

Con respecto a los resultados, estos consideran, en primer lugar, que el gasto en cultura es demasiado bajo, debido a que se puede considerar que su valor sea más simbólico que monetario. En segundo lugar, la determinación de una tasa de interés para la cultura por bajo de la del mercado crediticio, por lo que debe considerarse una política de subsidios gubernamentales para otorgar intereses especiales a este sector.

Por otro lado, el papel de la cultura en la economía reviste una importancia tal que iguala al total del sector primario, lo cual conlleva a tener niveles de participación por encima de la agricultura.

Estos resultados también implican no sólo una desigualdad en la brecha económica, sino también en la brecha cultural, pues el gasto en cultura se debe a diversos factores, más allá de los monetarios. De ahí, la poca disposición a pagar sobre bienes y servicios culturales.

Con respecto a la tasa de interés propia para el sector, implica poner atención sobre los posibles rendimientos que se pueden para aquellos que invierten en las ediciones de libros, así como las producciones, presentaciones y/o exposiciones de los productos que los mismos artistas ponen a disposición del público.

Por último, el desarrollo de políticas públicas culturales debe tener un fundamento económico que permita su fomento, tanto en términos de creación, como de generación de un público que esté en la disposición de adquirir los bienes y servicios culturales.

\section{REFERENCIAS}

Aguado Quintero, L. F., Palma, L. y Pulido Pavón, N. (2017). 50 años de economía de la cultura. Explorando sus raíces en la historia del pensamiento económico. Cuadernos de economía, 36(70), 197-225. https://doi.org/10.15446/cuad.econ.v36n70.53813 
Banco Mundial (s.f.) Indicadores del Desarrollo Mundial. Recuperado de https://databank.bancomundial.org/data/source/world-development-indicators

Batista Medina, J. A. (2006). Economía Cultural: elementos para un análisis cultural de lo económico y para una crítica de la Economía (ortodoxa). Revista Porik an, (11), 123-155. Recuperado de http://www.unicauca.edu.co/porik an/imagenes 3noanteriores/No.11porikan/articulo3.pdf

Blanchard, O., Amighini, A. \& Giavazzi, F. (2012), Macroeconomía. 5a. Ed., Madrid: Editorial Pearson.

Bourdieu, P. (2011). Las estrategias de la reproducción social. Buenos Aires: Siglo XXI.

Conaculta (2010), Encuesta Nacional de Hábitos, Prácticas y Consumo Culturales. Recuperado de https://www.cultura.gob.mx/recursos/banners/ENCUESTA NACIONAL.pdf

Cooter, R. D. \& Ulen, T. (2016). Derecho y economía. Recuperado de https://bit.ly/2zbGeJT

Dornbusch, R., Fischer, S. \& Startz, R. (2004), Macroeconomía. 9a. Ed. Bogotá: Editorial McGraw Hill.

Elizondo, R. (2012). Estimaciones del PIB Mensual Basadas en el IGAE. Recuperado de https://bit.ly/2P6svPy

INEGI (2018a). Banco de Información Económica. Indicadores Económicos de Coyuntura. Recuperado de https://www.inegi.org.mx/sistemas/bie/

INEGI (2018b). Sistema de Cuentas Satelitales de la Cultura. Tabulados. Recuperado de https://www.inegi.org.mx/programas/cultura/2013/default.html\#Tabulados

Guzmán Cárdenas, C. E. (2003). La Cultura suma. Las relaciones entre economía y cultura. En Seminario Internacional sobre Indicadores Culturales: su contribución al estudio de la economía y la cultura. Ciudad de México, México. Recuperado de https://sic.cultura.gob.mx/documentos/814.pdf

Molina Roldán, A. (2016). La construcción del sentido social en la gestión cultural en América Latina. International Journal of Arts Management, 44-55. Recuperado de https://bit.ly/2NppJ5k

Monasterio Astobiza, A. (2017). ¿Qué es cultura en la «economía de la cultura»? Definiendo la cultura para crear modelos mensurables en economía cultural. Arbor, 193(783), 1-10. http://dx.doi.org/10.3989/arbor.2017.783n1007

Palma, L. A. \& Aguado, L. F. (2010), Economía de la Cultura. Una nueva área de especialización de la economía. Revista de Economía Institucional, 12(22), 129-165. Recuperado de https://revistas.uexternado.edu.co/index.php/ecoins/article/view/386

Throsby, D. (2001). Economía y Cultura. Madrid: Cambridge University Press. 
Unesco (2013), Informe sobre la economía creativa Edición especial 2013. Ampliar los cauces de desarrollo local. Recuperado de http://www.unesco.org/culture/pdf/creative-economyreport-2013-es.pdf

Unesco (2014). Indicadores Unesco de cultura para el desarrollo. Manual metodológico. Recuperado de https://es.unesco.org/creativity/sites/creativity/files/iucd manual metodologico 1.pdf

Yudice, G. (2002). El recurso de la cultura (usos de la cultura en la era global). Barcelona: Gedisa. 